\title{
A perspetiva dos médicos de família portugueses quanto à referenciação de crianças com problemas de comunicação e linguagem
}

Inês Dinis, ${ }^{1}$ Fátima Maia, ${ }^{2}$ Rute F. Meneses, ${ }^{3}$ Rafael Sousa ${ }^{4}$

\section{RESUMO}

Objetivos: Descrever o perfil do médico de família em Portugal, no que concerne à referenciação de crianças com problemas de comunicação e linguagem e identificar barreiras ao processo de referenciação.

Tipo de estudo: Observacional, transversal e descritivo.

Local: Região Centro, Lisboa e Vale do Tejo, Região Norte e Região Autónoma dos Açores.

População: Médicos de família da região Centro, Lisboa e Vale do Tejo, região Norte e Região Autónoma dos Açores.

Métodos: Construção e aplicação de um questionário on-line, com 35 questões com resposta em escala de Likert (0-5) e uma questão semiaberta. O questionário foi enviado a nível nacional, segundo um método de amostragem por redes. Foi assegurado o anonimato e a confidencialidade dos dados.

Resultados: Participaram 55 médicos de família. Cerca de $40 \%$ dos inquiridos consideraram-se bastante satisfeitos com o processo de referenciação; $55 \%$ manifestaram-se pouco ou nada satisfeitos com a aprendizagem de conteúdos acerca do desenvolvimento de comunicação e linguagem durante o curso de medicina; $58 \%$ referiram que o encaminhamento para o Sistema Nacional de Intervenção Precoce na Infância ocorre com pouca ou nenhuma frequência. Foram identificadas seis barreiras ao processo de referenciação: constrangimentos com serviços; recursos humanos; burocracia; lentidão do processo; nível de conhecimentos relacionados com o processo de referenciação; atitude dos pais.

Conclusão: Os resultados do estudo não permitem descrever o perfil do médico de família em Portugal, pelo que deve ser considerado um estudo piloto. Contudo, são indicativos da perspetiva dos médicos participantes acerca da referenciação de crianças com problemas de comunicação e linguagem, bem como das barreiras associadas a este processo.

Palavras-chave: Problemas de comunicação e linguagem; Processo de referenciação; Médico de família; Intervenção precoce; Terapeuta da fala.

\section{INTRODUÇÃO}

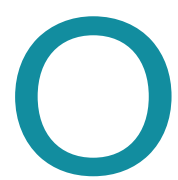

s problemas de comunicação e linguagem (PCL) referem-se a um variado espectro de problemas, incluindo perturbações específicas de linguagem, perturbações dos sons da fala, perturbações da fluência, atrasos de de-

1. Universidade Fernando Pessoa. Agrupamento de Escolas da Sé, Guarda

2. Universidade Fernando Pessoa (FCS/ESS)

3. Universidade Fernando Pessoa (FCHS/CTEC/FP-B2S/HE)

4. USF Infante D. Henrique, ACeS Dão-Lafões. senvolvimento de linguagem/emergência tardia de linguagem, entre outros, que limitam a aquisição e desenvolvimento da linguagem e da comunicação. Em idade pré-escolar a maior parte dos estudos aponta para uma prevalência entre 2,3\% e 19\%.1.-5 Em Portugal, um estudo refere uma prevalência de $14,9 \% .{ }^{6}$ Estes problemas podem prejudicar o desenvolvimento de competências sociais, académicas e emocionais do indivíduo ao longo da sua vida. ${ }^{7-10}$ Já pelos três anos de idade é possível constatar que estas crianças apresentam 
índices de qualidade de vida mais baixos. ${ }^{9} \mathrm{Na}$ adolescência, cerca de $40 \%$ dos indivíduos com perturbação específica de linguagem revelam dificuldades de interação com os pares. ${ }^{8,10}$ Para além disso, muitas das vezes a ocorrência de um problema deste tipo gera preocupação e stress na família. ${ }^{11}$ Estas situações podem também ter implicações futuras negativas para a sociedade. ${ }^{3,12-14}$ Nos Estados Unidos da América um estudo verificou que o desemprego atinge $75 \%$ das pessoas com problemas graves de fala. ${ }^{14}$ Por outro lado, a intervenção precoce nestes casos tem-se revelado benéfica, eficaz e com boa relação custo-benefício. ${ }^{15-16}$

Os médicos de família encontram-se numa posição privilegiada para detetar e referenciar precocemente estes problemas. ${ }^{4-5,17-19}$ O Plano Nacional de Saúde Infantil e Juvenil (PNSIJ), documento base para as consultas de vigilância de saúde infantil, recomenda a referenciação destas situações para o Sistema Nacional de Intervenção Precoce na Infância (SNIPI).$^{20}$ Este sistema foi consagrado em Portugal em 2009, com a publicação do Decreto-Lei n. ${ }^{\circ}$ 281/2009, de 6 de outubro, e pretende abranger todas as famílias e crianças com alterações nas funções ou estruturas do corpo ou com risco grave de atraso de desenvolvimento, nas quais se incluem os problemas de comunicação e linguagem. ${ }^{21}$ Todavia, alguns estudos apontam para um reduzido encaminhamento de crianças com problemas de desenvolvimento para serviços de intervenção precoce, inclusive em Portugal. ${ }^{22-27}$ Para além disso, há poucos estudos que tentaram compreender quais os motivos para esta situação e não é conhecido nenhum em Portugal. ${ }^{17,22}$

Assim, a finalidade desta investigação foi compreender qual a perspetiva dos médicos de família relativamente à referenciação precoce de crianças com problemas de comunicação e linguagem e saber quais as barreiras que estes identificam no processo de referenciação. Definiram-se dois objetivos gerais: descrever o perfil do médico de família em Portugal, no que concerne à referenciação de crianças com problemas de comunicação e linguagem; e identificar barreiras ao processo de referenciação.

\section{MÉTODOS}

O estudo apresentado é observacional, transversal e descritivo. Construiu-se um questionário on-line na plataforma FORMULÁRIOS DO GOOGLE, onde constava uma car- ta introdutória e um consentimento informado. O questionário apresentava sete partes com 35 questões fechadas com opções de resposta em escala de Likert de cinco níveis e uma questão semiaberta, distribuídas da seguinte forma: I. Caracterização socioprofissional; II. Satisfação com fatores internos e externos necessários à referenciação de crianças com problemas de comunicação e linguagem [opções: 1) Nada satisfeito(a); 2) Pouco satisfeito(a);3) Satisfeito(a); 4) Bastante satisfeito(a); 5) Muito satisfeito(a)]; III. Frequência de comportamentos durante a consulta de saúde infantil (opções: 1) Nunca ocorre; 2) Ocorre pouco;3) Ocorre com alguma frequência; 4) Ocorre com bastante frequência; 5) Ocorre com muita frequência]; IV. Importância atribuída a atitudes, comportamentos e situações relativamente ao processo de deteção e encaminhamento de crianças com problemas de comunicação e linguagem [opções: 1) Nada importante, 2) Pouco importante, 3) Importante, 4) Bastante importante, 5) Muito importante];V. Fatores que influenciam o encaminhamento [opções: 1) Não influencia; 2) Influencia pouco; 3) Influencia; 4) Influencia bastante; 5) Influencia muito]; VI. Interesse pessoal relativo ao desenvolvimento de conhecimentos sobre comunicação e linguagem [opções: 1) Nada interessado(a); 2) Pouco interessado(a); 3) Interessado(a); 4) Bastante interessado(a); 5) Muito interessado(a)]. A parte VII - «Satisfação com o processo de referenciação atual de crianças com problemas de comunicação e linguagem» - incluía uma questão fechada (opções: 1) Nada satisfeito(a); 2) Pouco satisfeito(a); 3) Satisfeito(a); 4) Bastante satisfeito(a); 5) Muito satisfeito(a)] e uma semiaberta - «No caso de considerar que o processo de referenciação não ocorre da forma mais eficiente, por favor indique três barreiras».

Foi realizado um pré-teste a três médicos de família e, consequentemente, foram revistas algumas questões para melhorar a compreensão das mesmas. Quanto à fiabilidade determinou-se que a consistência interna do questionário ( $\alpha$ de Cronbach) é boa $(\alpha=0,8){ }^{28}$

O questionário foi enviado entre novembro de 2015 e maio de 2016, segundo um método não probabilístico de amostragem por redes, ou seja, com a colaboração de quatro médicos de família selecionados (um da região Centro, um de Lisboa e Vale do Tejo, um da região Norte e um da Região Autónoma dos Açores).

Os dados quantitativos foram tratados com recurso ao programa Statistical Package for the Social Sciences 


\begin{tabular}{|c|c|c|c|}
\hline \multirow{2}{*}{\multicolumn{2}{|c|}{ Varáveis sociodemográficas }} & \multicolumn{2}{|c|}{ Frequências } \\
\hline & & $n$ & $\%$ \\
\hline \multirow[t]{2}{*}{ Sexo } & Feminino & 41 & 74,5 \\
\hline & Masculino & 14 & 25,5 \\
\hline \multirow[t]{4}{*}{ Local } & Centro & 20 & 36,4 \\
\hline & Norte & 6 & 10,9 \\
\hline & Lisboa e Vale do Tejo & 23 & 41,8 \\
\hline & Região Autónoma dos Açores & 6 & 10,9 \\
\hline \multirow[t]{2}{*}{ Instituição } & USF & 49 & 89,1 \\
\hline & ACeS & 6 & 10,9 \\
\hline \multirow[t]{2}{*}{ Condição profissional } & Interno & 31 & 56,4 \\
\hline & Especialista & 24 & 43,6 \\
\hline \multirow{6}{*}{$\begin{array}{l}\text { Anos de experiência } \\
\text { profissional }\end{array}$} & 1 a 2 & 12 & 21,8 \\
\hline & 3 a 4 & 22 & 40,0 \\
\hline & 5 a 10 & 10 & 18,2 \\
\hline & 11 a 20 & 3 & 5,50 \\
\hline & Mais de 20 & 8 & 14,5 \\
\hline & \multicolumn{3}{|l|}{$\begin{array}{l}\text { Média total: } 8,04( \pm 9,6) \\
\text { Amplitude de anos de experiência: de } 1 \text { a } 33\end{array}$} \\
\hline
\end{tabular}

liação destes problemas pelo terapeuta da fala é muito importante.

Quanto à valorização de atitudes de deteção e referenciação precoces (Quadro III), o encaminhamento de crianças com suspeita de problemas de comunicação e linguagem dos três aos seis anos de idade foi considerado muito importante por mais médi$\cos (71 \%)$ do que dos 0 aos 36 meses (26\%). Por outro lado, mais médicos assinalaram como muito importante a atitude «observar, detetar precocemente e encaminhar precocemente» (56\%) do que a atitude «observar, suspeitar e esperar» (13\%). Quase metade destes médicos $(45 \%)$ consideraram ainda que seria muito importante a existência de rastreios direcionados para a deteção de problemas de comunicação e linguagem nos

- IBM® SPSS ${ }^{\circledR}$ for Windows. Para os dados qualitativos recorreu-se à análise de conteúdo, na qual a unidade de registo foi o tema. As categorias e subcategorias emergiram dos resultados e a análise foi validada por outro codificador, especialista na área.

\section{RESULTADOS}

Cinquenta e cinco médicos de família participaram no estudo. A maioria dos participantes é do sexo feminino $(74,5 \%)$ e trabalha em Unidades de Saúde Familiar $(89,1 \%)$. Quanto aos locais, a maior parte é de Lisboa e Vale do Tejo $(41,8 \%)$ e da região Centro $(36,4 \%)$. Aproximadamente $56 \%$ são médicos internos e $43 \%$ são especialistas. A média de experiência profissional é de oito anos (Quadro I).

Apresentar-se-ão, em primeiro lugar, os resultados quantitativos.

Relativamente à valorização dos intervenientes profissionais no processo de referenciação de crianças com problemas de comunicação e linguagem (Quadro II), $60 \%$ dos inquiridos consideraram o seu papel muito importante. Para além disso, 70\% referiram valorizar muito o trabalho de equipa e $58 \%$ afirmaram que a ava- cuidados de saúde primários.

No que concerne aos procedimentos para a deteção e referenciação deste tipo de problemas (Quadro IV), $55 \%$ dos médicos referiram utilizar com muita frequência a Escala de Mary Sheridan Modificada (EADMSM), enquanto 53\% declararam nunca utilizar ou utilizar pouco o Modified Checklist for Autism in Toddlers (M-CHAT). Alguns dos inquiridos (38\%) afirmaram que o encaminhamento destas crianças para o hospital ocorre com bastante frequência. Contudo, 36\% dos mesmos declarou que o encaminhamento para o SNIPI ocorre com pouca frequência e $22 \%$ referiram nunca o ter feito.

Em relação à satisfação com o processo de deteção e referenciação (Quadro V), 42\% dos médicos consideraram-se bastante satisfeitos com o processo de referenciação e $74 \%$ referiram estar satisfeitos ou bastante satisfeitos com os seus conhecimentos sobre o mesmo. Cerca de metade (49\%) referiu estar satisfeita com este tipo de informação no PNSIJ e 31\% julgaram-se pouco satisfeitos.

Quanto à satisfação com os conhecimentos acerca do desenvolvimento de comunicação e linguagem (Quadro VI), 62\% dos inquiridos consideraram-se 


\begin{tabular}{|c|c|c|c|c|c|}
\hline \multirow[b]{2}{*}{ Questões } & \multicolumn{5}{|c|}{ Classificação dos médicos de família } \\
\hline & $\begin{array}{c}1 \\
\text { (Nada } \\
\text { importante) }\end{array}$ & $\begin{array}{c}2 \\
\text { (Pouco } \\
\text { importante) }\end{array}$ & $\begin{array}{c}3 \\
\text { (Importante) }\end{array}$ & $\begin{array}{c}4 \\
\text { (Bastante } \\
\text { importante) }\end{array}$ & $\begin{array}{c}5 \\
\text { (Muito } \\
\text { importante) }\end{array}$ \\
\hline Encaminhamento dos 0 aos 36 meses & $0 \%$ & $9 \%$ & $29 \%$ & $36 \%$ & $26 \%$ \\
\hline Encaminhamento dos 3 aos 6 anos & $0 \%$ & $0 \%$ & $2 \%$ & $27 \%$ & $71 \%$ \\
\hline $\begin{array}{l}\text { Atitude «observar, detetar precocemente e } \\
\text { encaminhar precocemente» }\end{array}$ & $0 \%$ & $2 \%$ & $13 \%$ & $29 \%$ & $56 \%$ \\
\hline Atitude «observar, suspeitar e esperar» & $2 \%$ & $9 \%$ & $31 \%$ & $45 \%$ & $13 \%$ \\
\hline Rastreios de PCL nos CSP & $0 \%$ & $6 \%$ & $9 \%$ & $40 \%$ & $45 \%$ \\
\hline
\end{tabular}

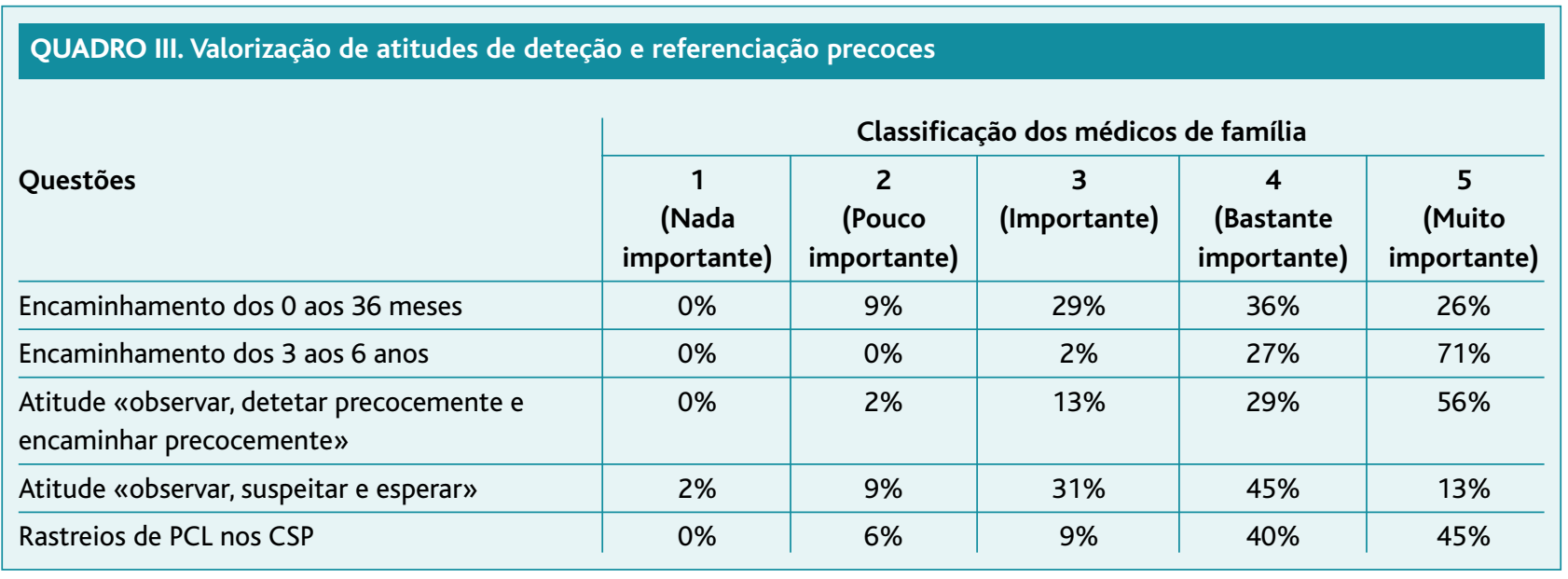

\begin{tabular}{|c|c|c|c|c|c|}
\hline \multirow[b]{2}{*}{ Questões } & \multicolumn{5}{|c|}{ Classificação dos médicos de família } \\
\hline & $\begin{array}{c}1 \\
\text { (Nunca ocorre) }\end{array}$ & $\begin{array}{c}2 \\
\text { (Ocorre pouco) }\end{array}$ & $\begin{array}{c}3 \\
\text { (Ocorre com } \\
\text { alguma } \\
\text { frequência) }\end{array}$ & $\begin{array}{c}4 \\
\text { (Ocorre com } \\
\text { bastante } \\
\text { frequência) }\end{array}$ & $\begin{array}{c}5 \\
\text { (Ocorre com } \\
\text { muita } \\
\text { frequência) }\end{array}$ \\
\hline Utilização da EADMSM & $4 \%$ & $7 \%$ & $9 \%$ & $25 \%$ & $55 \%$ \\
\hline Utilização do M-Chat & $29 \%$ & $24 \%$ & $29 \%$ & $9 \%$ & $9 \%$ \\
\hline Encaminhamento para o hospital & $0 \%$ & $24 \%$ & $27 \%$ & $38 \%$ & $11 \%$ \\
\hline Encaminhamento para o SNIPI & $22 \%$ & $36 \%$ & $27 \%$ & $13 \%$ & $2 \%$ \\
\hline
\end{tabular}

satisfeitos com os mesmos. Quarenta por cento manifestaram-se satisfeitos com a disponibilização de formação adicional sobre comunicação e linguagem durante a especialização/carreira em medicina geral e fa- miliar. Todavia, $44 \%$ declaram-se pouco ou nada satisfeitos. Mais de metade destes médicos (55\%) assinalou que estava pouco ou nada satisfeita com a aprendizagem destes conteúdos durante o curso de medicina. 


\begin{tabular}{|c|c|c|c|c|c|}
\hline \multirow[b]{2}{*}{ Questões } & \multicolumn{5}{|c|}{ Classificação dos médicos de família } \\
\hline & $\begin{array}{c}1 \\
\text { (Nada } \\
\text { satisfeito) }\end{array}$ & $\begin{array}{c}2 \\
\text { (Pouco } \\
\text { satisfeito) }\end{array}$ & $\begin{array}{c}3 \\
\text { (Satisfeito) }\end{array}$ & $\begin{array}{c}4 \\
\text { (Bastante } \\
\text { satisfeito) }\end{array}$ & $\begin{array}{c}5 \\
\text { (Muito } \\
\text { satisfeito) }\end{array}$ \\
\hline Processo de referenciação & $2 \%$ & $20 \%$ & $36 \%$ & $42 \%$ & $0 \%$ \\
\hline $\begin{array}{l}\text { Conhecimentos sobre o processo } \\
\text { de referenciação }\end{array}$ & $2 \%$ & $13 \%$ & $43 \%$ & $31 \%$ & $11 \%$ \\
\hline Informações do PNSIJ & $9 \%$ & $31 \%$ & $49 \%$ & $11 \%$ & $0 \%$ \\
\hline
\end{tabular}

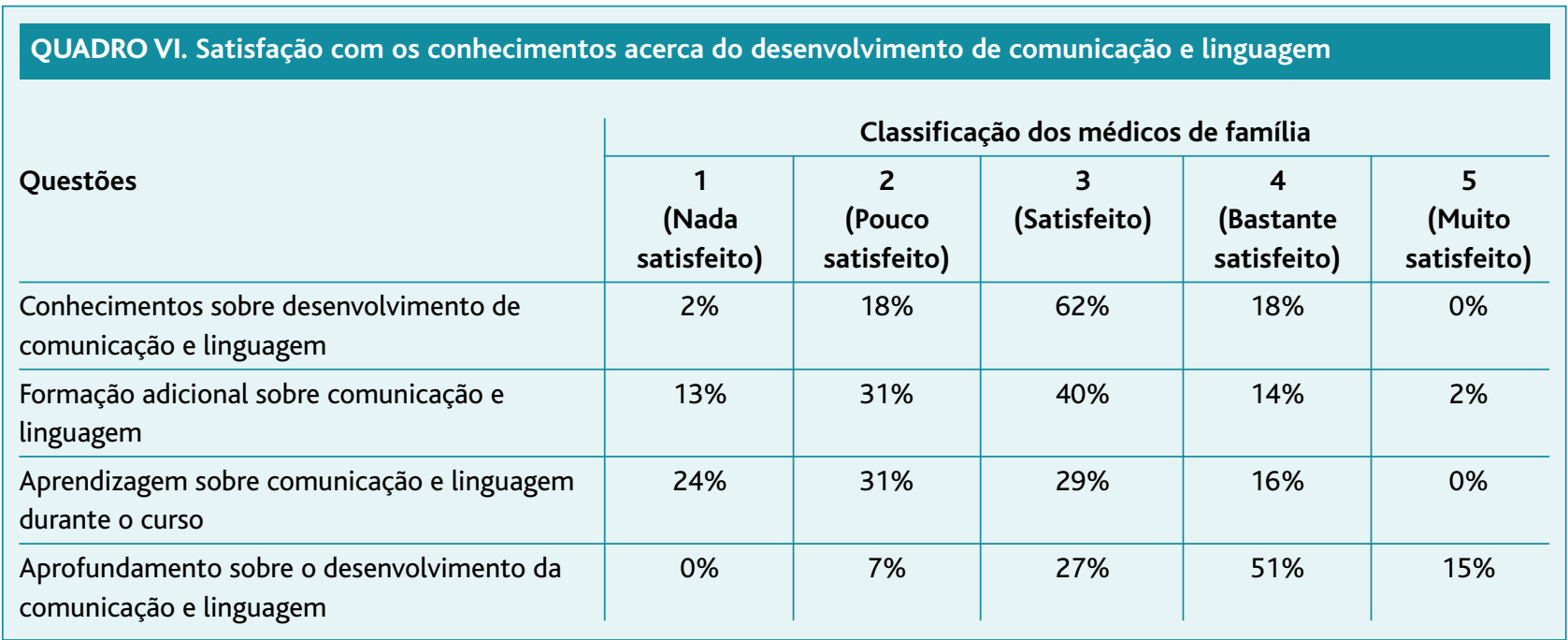

\begin{tabular}{|c|c|c|c|c|c|}
\hline \multirow[b]{2}{*}{ Questões } & \multicolumn{5}{|c|}{ Classificação dos médicos de família } \\
\hline & $\begin{array}{c}1 \\
\text { (Não } \\
\text { influencia) }\end{array}$ & $\begin{array}{c}2 \\
\text { (Influencia } \\
\text { pouco) }\end{array}$ & $\begin{array}{c}3 \\
\text { (Influencia) }\end{array}$ & $\begin{array}{c}4 \\
\text { (Influencia } \\
\text { bastante) }\end{array}$ & $\begin{array}{c}5 \\
\text { (Influencia } \\
\text { muito) }\end{array}$ \\
\hline Preocupação dos pais & $0 \%$ & $0 \%$ & $20 \%$ & $53 \%$ & $27 \%$ \\
\hline Satisfação com os serviços & $0 \%$ & $6 \%$ & $27 \%$ & $45 \%$ & $22 \%$ \\
\hline Resultados da EADMSM & $0 \%$ & $9 \%$ & $13 \%$ & $56 \%$ & $22 \%$ \\
\hline Resposta dos serviços & $5 \%$ & $33 \%$ & $25 \%$ & $35 \%$ & $2 \%$ \\
\hline Eficácia da EADMSM & $2 \%$ & $20 \%$ & $51 \%$ & $24 \%$ & $3 \%$ \\
\hline
\end{tabular}

Cerca de metade $(51 \%)$ demonstrou-se bastante interessada em aprofundar conhecimentos nesta área.

No que respeita aos potenciais fatores de influência na tomada de decisão para a referenciação (Quadro
VII), todos os médicos consideraram que a preocupação dos pais com o desenvolvimento de comunicação e linguagem dos filhos influencia a sua tomada de decisão para a referenciação. Quarenta e cinco por cento 


\begin{tabular}{|c|c|c|c|}
\hline Categorias & Subcategorias & $\begin{array}{l}\text { Frequência de } \\
\text { referências }\end{array}$ & Total \\
\hline \multirow[t]{5}{*}{ Constrangimentos com serviços } & Tempo de espera (resposta/processo) & 8 & 15 \\
\hline & Articulação de serviços e profissionais & 3 & \\
\hline & Distância entre serviços & 1 & \\
\hline & Financeiros & 2 & \\
\hline & Capacidade de resposta & 1 & \\
\hline \multirow[t]{4}{*}{ Recursos humanos } & Falta de TF nos CSP & 4 & 10 \\
\hline & Falta de TF nos serviços de saúde e educativos & 2 & \\
\hline & Poucos recursos & 3 & \\
\hline & Profissionais envolvidos no processo & 1 & \\
\hline Burocracia & Burocracia & 2 & 2 \\
\hline Lentidão do processo & Lentidão do processo & 1 & 1 \\
\hline \multirow{2}{*}{$\begin{array}{l}\text { Conhecimentos relacionados com o processo de } \\
\text { referenciação }\end{array}$} & Desconhecimento & 2 & 4 \\
\hline & Falta de formação (curso/internato) & 2 & \\
\hline Atitude dos pais & Insegurança e desvalorização da referenciação & 2 & 2 \\
\hline
\end{tabular}

dos inquiridos manifestaram que a sua satisfação com os serviços que apoiam as crianças com estes problemas influencia bastante a decisão para a referenciação. Neste sentido, 35\% assinalaram bastante satisfação com os mesmos, embora $33 \%$ se tenham declarado pouco satisfeitos. Mais de metade $(56 \%)$ considerou que os resultados da Escala de Mary Sheridan Modificada influenciam bastante a decisão de referenciação e cerca de metade (51\%) declarou-se satisfeita com este instrumento de avaliação.

Apresentar-se-ão seguidamente os resultados qualitativos.

Dos 55 médicos que responderam ao questionário, $16(29,1 \%)$ apontaram barreiras ao processo de referenciação. Como se verificar no Quadro VIII, as respostas obtidas dividiram-se em seis grandes categorias e 14 subcategorias. Posteriormente serão apresentadas algumas declarações exemplificativas efetuadas pelos médicos de família.

No total, os constrangimentos com os serviços foram os mais mencionados. Neste âmbito, a barreira referida com mais frequência foi o tempo de espera para a obtenção de uma resposta após referenciação: "atraso no atendimento pelos cuidados hospitalares das situa- ções referenciadas" (sujeito 20). Também foram apontados problemas com a articulação entre serviços e profissionais e com a distância entre serviços: "ausência de feedback/comunicação entre os serviços” (sujeito 47); "falta de ligação entre terapeutas da fala, médicos, professores e família" (sujeito 32); "distância dos locais de prestação de cuidados do local onde presto cuidados" (sujeito 20). Para além disso, foram identificadas barreiras financeiras relacionadas com os elevados custos das intervenções fora do sistema público em comparação com os rendimentos das famílias: "terapia da fala feita no privado é cara para a maioria das famílias" (sujeito 44). A capacidade de resposta deficitária dos serviços também foi apontada: "o hospital da minha área (...) não tem capacidade de resposta" (sujeito 44). Nos relatos analisados foi evidente que a maioria destes constrangimentos é relativa ao hospital. Na articulação entre serviços também são mencionados os serviços escolares. Os serviços do SNIPI nunca foram referidos neste contexto.

Os problemas com recursos humanos foram os segundos mais mencionados. A ausência de terapeutas da fala, tanto nos cuidados de saúde primários como noutros serviços de saúde e educativos, também foram bas- 
tante referidos: "a referenciação é feita ao hospital de referência em que as terapeutas da fala apenas fazem a avaliação diagnóstica. Depois não há capacidade de resposta (pela falta de pessoal) para fazer o acompanhamento terapêutico" (sujeito 27); "necessidade de terapeuta da fala no ACeS (...), de forma a melhorar a articulação MGF/TF" (sujeito 4); "não existir terapia da fala nos cuidados primários para avaliação dos casos em que haja dúvidas e acompanhamento daqueles que necessitarem" (sujeito 46). A falta de recursos humanos e os profissionais envolvidos no processo também foram consideradas barreiras: "a falta de terapeutas da fala ou outros profissionais relacionados, integrados nas equipas multidisciplinares dos CS. É ao nível dos serviços privados que estes profissionais se encontram com mais frequência" (sujeito 50).

A burocracia e a lentidão do processo também foram barreiras identificadas pelos médicos desta amostra: "burocracia" (sujeito 18); "lentidão do processo" (sujeito 53).

Outra das categorias encontrada é alusiva aos conhecimentos relacionados com o processo de referenciação, nomeadamente desconhecimento e falta de formação durante o curso ou internato: "ausência ou desconhecimento de critérios de referenciação bem definidos e disponíveis" (sujeito 13); "formação deficitária" (sujeito 18).

A insegurança e a desvalorização dos pais face à referenciação, devido ao receio de estigmatização e ao desconhecimento do impacto dos problemas de linguagem e comunicação, respetivamente, também foram consideradas uma barreira: "pais renitentes à referenciação por medo à estigmatização" (sujeito 33); "muitos pais não compreendem que as alterações da linguagem terão impacto na criança, não só a nível de estudos, mas também de socialização e outros" (sujeito 44$)$.

\section{DISCUSSÃO}

Apesar de não ter sido foi possível definir o perfil do médico de família em Portugal, dada a caracterização da amostra e o método de recolha de dados, foi possível recolher algumas perceções dos médicos participantes acerca da referenciação de crianças com problemas de comunicação e linguagem e das barreiras existentes ao processo, que se descrevem seguidamente.
A maioria dos médicos de família da amostra pareceu valorizar o seu papel, a avaliação feita pelo terapeuta da fala e o trabalho de equipa relativamente ao processo de referenciação de crianças com problemas de comunicação e linguagem, o que vai ao encontro da literatura. . $^{426,29-31}$

A maioria destes médicos pareceu valorizar a deteção e o encaminhamento precoces, o que se destacou na maior importância atribuída à atitude «observar, detetar precocemente e encaminhar precocemente». Todavia, pareceu haver uma desvalorização deste comportamento na faixa etária dos 0 aos 36 meses, face à faixa etária dos três aos seis anos de idade. Estes resultados estão de acordo com a literatura, na qual é descrita uma tendência para um encaminhamento mais tardio. ${ }^{17,32-33}$ Contudo, as vantagens e importância de uma intervenção o mais precoce possível, após a deteção do problema, são cada vez mais conhecidas e defendidas. ${ }^{15,34-37}$

Relativamente aos procedimentos, os inquiridos pareceram utilizar pouco o M-CHAT, embora a sua aplicação seja recomendada no PNSIJ entre os 16 e os 30 meses. ${ }^{20}$ Esta situação poderá decorrer da falta de formação para a aplicação do mesmo. ${ }^{38}$ Numa outra investigação portuguesa, uma das dificuldades sentidas por $37 \%$ dos médicos prendia-se precisamente com a formação insuficiente para a aplicação das escalas de avaliação. ${ }^{43}$ Considera-se que o M-CHAT poderia ser um instrumento útil e rápido na deteção precoce de problemas de desenvolvimento, como o autismo e outros relacionados com a comunicação. Por outro lado, a maioria dos médicos referiu utilizar a Escala de Mary Sheridan Modificada para a deteção de problemas de comunicação e linguagem. Efetivamente, segundo a literatura, os testes informais baseados em marcos de desenvolvimento são os mais utilizados em contexto de cuidados de saúde primários..$^{20,32,38-42}$ Os resultados desta escala parecem influenciar a maior parte destes médicos para a decisão de encaminhamento. Contudo, cerca de metade demonstrou-se apenas satisfeita com este instrumento e $20 \%$ afirmou-se pouco satisfeita, o que, no entendimento dos autores do presente estudo, pode originar relutância quanto à referenciação destas crianças. Também pode dar-se o caso destes médicos privilegiarem a perceção clínica e os sinais de alarme, como já foi indicado num outro estudo português acer- 
ca do estabelecimento de critérios de encaminhamento para os cuidados de saúde secundários..$^{43}$ Assim, poderá haver uma maior confiança no juízo clínico do que nos resultados de um teste no momento de referenciar.

A generalidade destes médicos de família declarou referenciar crianças com problemas de comunicação e linguagem para o hospital. Por outro lado, as referenciações para o SNIPI foram mencionadas com bastante menor frequência. Este é um resultado esperado, uma vez que, de acordo com a literatura, há uma quantidade reduzida de referenciações provenientes dos cuidados de saúde primários para os serviços de intervenção precoce. ${ }^{22-25,27}$ Apesar de a maioria dos médicos do presente estudo ter referido que está satisfeita com os conhecimentos acerca do processo de referenciação, esta situação pode indicar algum desconhecimento, uma vez que o encaminhamento destas crianças para o SNIPI é uma das recomendações do PNSIJ. ${ }^{20}$

Apesar dos inquiridos terem referido, globalmente, que estão satisfeitos com o processo de referenciação e com os seus conhecimentos sobre o mesmo, uma das barreiras identificadas prendia-se com a falta de conhecimento acerca do processo. A literatura indica que existe algum desconhecimento sobre o processo de referenciação por parte dos médicos que exercem funções nos cuidados de saúde primários. ${ }^{19,22}$

A maior parte dos médicos desta amostra considerou-se satisfeita com os seus conhecimentos sobre desenvolvimento de comunicação e linguagem. Todavia, apenas $18 \%$ assinalaram estar bastante satisfeitos e ninguém se declarou muito satisfeito. Num estudo realizado em Portugal, $50 \%$ dos médicos de família referiram ter dúvidas em relação ao que valorizar e quando referenciar durante a avaliação do desenvolvimento psicomotor. ${ }^{43}$ Pensa-se que este é um aspeto importante, uma vez que a satisfação com os próprios conhecimentos sobre o desenvolvimento infantil parece ser um fator que influencia a tomada de decisão para a referenciação. ${ }^{29}$ Do mesmo modo, cerca de metade considerou-se satisfeita com a informação disponível no PNSIJ e 31\% afirmaram-se pouco satisfeitos. Estes resultados são semelhantes aos encontrados noutro estudo, no qual $21 \%$ dos médicos consideraram que as orientações contidas neste plano sobre o desenvolvimento psicomotor não eram esclarecedoras. ${ }^{43}$
Acresce que cerca de metade dos inquiridos manifestou-se pouco ou nada satisfeita com a disponibilização de formação adicional sobre comunicação e linguagem durante a especialização/carreira em medicina geral e familiar, sendo que a maioria se revelou interessada em aprofundar conhecimentos nesta área. Noutros estudos, os médicos que trabalham em cuidados de saúde primários também têm manifestado este interesse. ${ }^{19,29}$ Aliás, a satisfação com a disponibilização de formação adicional sobre problemas de desenvolvimento já foi apontada como um fator que influencia positivamente a referenciação. ${ }^{29}$ Estes resultados poderão indicar que os médicos deste estudo poderão sentir-se mais desmotivados para referenciar devido à falta de formação/informação.

Outro aspeto menos positivo foi a satisfação com a aprendizagem de conteúdos relacionados com problemas de comunicação e linguagem durante o curso de medicina. Mais de metade dos médicos considerou-se pouco ou nada satisfeita. Para além disso, uma das barreiras à referenciação identificada foi a falta de formação durante o curso/internato. Num outro estudo concluiu-se que pediatras de cuidados de saúde primários, que frequentaram um curso de medicina com conteúdos relacionados com a área dos problemas de desenvolvimento, apresentavam um nível maior de satisfação com as suas competências profissionais a este nível. ${ }^{29}$ Deste modo, a sensação de insatisfação perante a aprendizagem destes conteúdos durante o curso poderá também prejudicar a tomada de decisão para o encaminhamento.

Todos os médicos assinalaram que são sensíveis à preocupação dos pais com o desenvolvimento dos filhos para a decisão de referenciação. Para além disso, uma das barreiras identificadas no processo de referenciação foi a insegurança e a desvalorização dos pais face a este procedimento. De facto, este aspeto tem sido apontado na literatura como um fator para os baixos índices de referenciação destas crianças. ${ }^{27,44}$

A satisfação com os serviços externos que acompanham crianças com problemas de comunicação e linguagem também parece influenciar o encaminhamento. No entanto, uma percentagem elevada declarou não estar muito satisfeita com as respostas existentes.

Considera-se que os motivos para este descontentamento podem estar relacionados com as duas maiores 


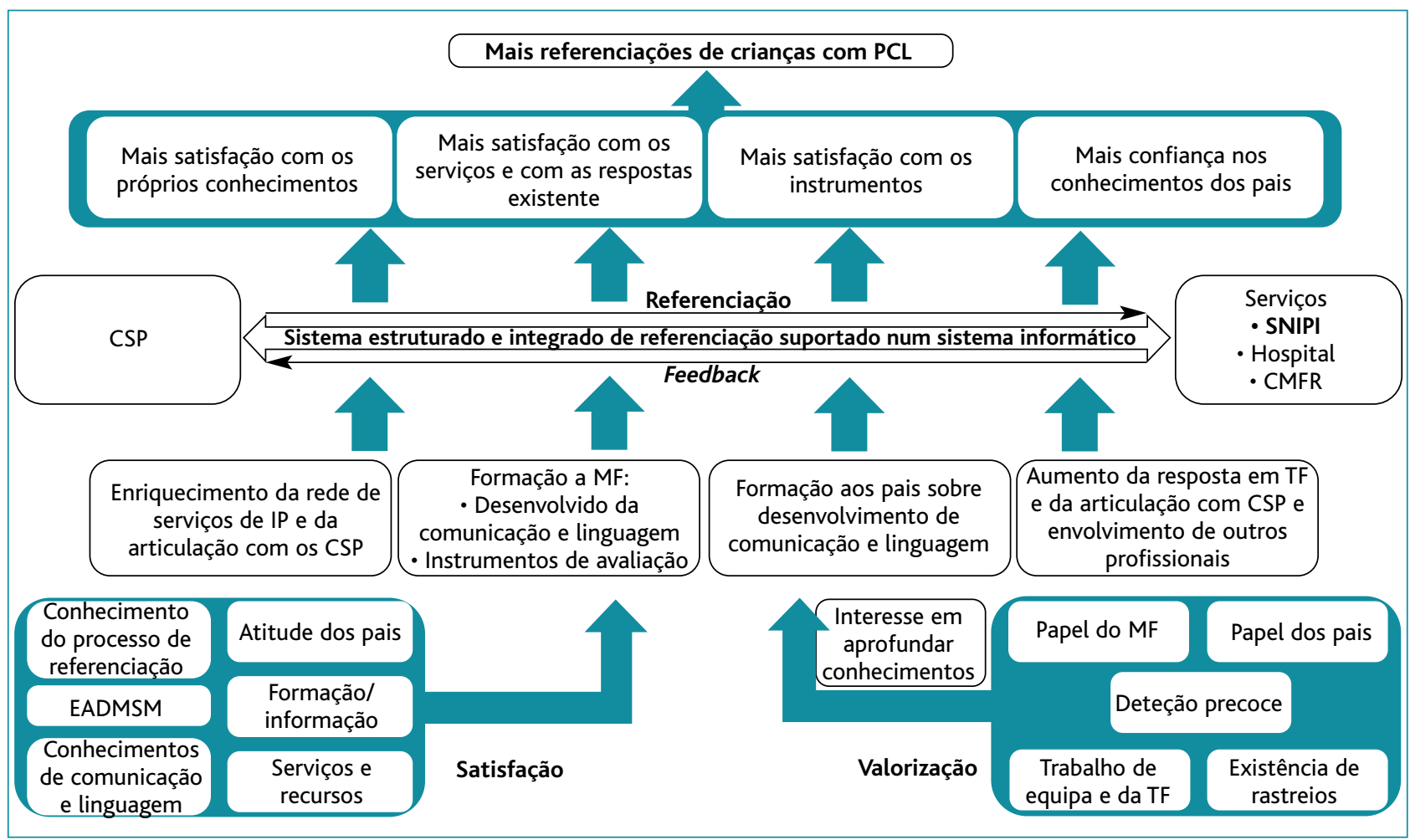

Figura 1. Esquema sugestivo de um sistema de referenciação de crianças com perturbação de comunicação e linguagem.

barreiras identificadas. Por um lado, os constrangimentos com os serviços, como o tempo elevado de resposta ou do processo e a falta de articulação entre serviços e profissionais. Por outro, os problemas com recursos humanos, como a falta de terapeutas da fala nos serviços de saúde e educativos. Na literatura, os médicos de cuidados de saúde primários referem que a ausência de outros profissionais de saúde durante a avaliação prejudica o processo de referenciação. ${ }^{26,32}$ De igual modo, a satisfação com os serviços que apoiam as crianças com problemas de desenvolvimento influencia a tomada de decisão para a referenciação. ${ }^{29}$ Assim, é possível que os médicos deste estudo se sintam condicionados por estes fatores, o que poderá limitar o número de crianças com problemas de comunicação e linguagem encaminhadas.

Os inquiridos identificaram ainda como barreiras ao processo de referenciação a burocracia e a lentidão. Segundo a literatura, a falta de um sistema estruturado de referenciação pode influenciar negativamente a tomada de decisão dos médicos. ${ }^{44}$ É possível que alguns mé- dicos de família desta amostra também se sintam limitados por este fator.

O conhecimento da perspetiva destes médicos de família e das barreiras que identificaram poderá contribuir para a melhoria do processo de referenciação de crianças com problemas de comunicação e linguagem. Neste sentido, elaborou-se um esquema sugestivo de algumas medidas que se consideram importantes para o desenvolvimento deste processo (Figura 1).

Das sugestões apresentadas destacam-se as seguintes:

- Enriquecimento da rede de serviços de intervenção precoce e da articulação com os cuidados de saúde primários;

- Formação dirigida a médicos de família sobre desenvolvimento de comunicação e linguagem e problemas associados e sobre os possíveis instrumentos de avaliação;

- Formação aos pais, em articulação com os cuidados de saúde primários, sobre comunicação e linguagem; 
- Aumento da resposta em terapia da fala e melhoria da articulação destes profissionais com os cuidados de saúde primários. Seria útil haver terapeutas da fala que estivessem inseridos nos cuidados de saúde primários e que pudessem facilitar a articulação entre serviços. O papel destes profissionais poderia alargar-se a outros contextos de atuação de terapia da fala, como os de deglutição e motricidade orofacial;

- Envolvimento de outros profissionais no processo de referenciação como, por exemplo, os enfermeiros. Todas estas sugestões poderiam ser integradas num sistema estruturado e integrado de referenciação, suportado preferencialmente por um sistema informático, de forma a aproximar os cuidados de saúde primários e os serviços externos. Para que o processo fosse mais facilitado seria aconselhável que as referenciações fossem efetuadas para o SNIPI, uma vez que este é um serviço especializado de intervenção precoce, constituído por equipas formadas por profissionais de diversas áreas $\mathrm{e}$ que contempla, teoricamente, uma abordagem transdisciplinar para problemas de desenvolvimento, como os problemas de comunicação e linguagem.

Este sistema integraria também uma componente de monitorização e acompanhamento das situações referenciadas. Os médicos de família teriam acesso ao feedback dos profissionais/serviços para onde encaminhassem as crianças com problemas de comunicação e linguagem. Desta forma, seria potencializada a articulação entre serviços e, consequentemente, a qualidade na prestação de cuidados a estas crianças e suas famílias.

Estas medidas poderiam contribuir para uma melhoria da satisfação dos médicos de família acerca dos seus conhecimentos sobre comunicação e linguagem, dos serviços e respostas existentes e dos instrumentos de avaliação. Considera-se que estas melhorias se traduziriam num aumento qualitativo das referenciações de crianças com problemas de comunicação e linguagem.

\section{CONCLUSÃO}

Este estudo apresenta algumas limitações. Por um lado, não foi possível definir o perfil do médico de família em Portugal, dado que se baseia numa amostra não probabilística, de pequena dimensão. Desta forma, não foi possível generalizar os resultados para a população portuguesa de médicos de família, que apresenta uma constituição diferente da estudada. Assim, considera-se que esse trabalho deve caracterizar-se como estudo piloto. Por outro lado, o questionário deveria apresentar melhores características psicométricas para a obtenção de resultados mais fiáveis.

Contudo, considera-se que este estudo levanta algumas questões pertinentes que merecem reflexão, nomeadamente no que respeita à formação acerca de problemas de comunicação e linguagem da criança no curso e internato de medicina, à necessidade de terapeutas da fala nos serviços e à articulação entre os cuidados de saúde primários e o SNIPI. Será importante um aprofundamento destas questões em investigações subsequentes.

Como sugestão de estudos futuros demonstra-se a pertinência dos dados obtidos neste trabalho, justificando a sua replicação e recorrendo a uma amostra probabilística, de maior dimensão e com maior abrangência geográfica, para obtenção de resultados com representatividade estatística que permitam conhecer a população estudada. Para além disso, devido a questões éticas, não foi possível analisar os dados de acordo com as regiões. Seria importante perceber se a região onde trabalham os médicos influencia a sua perspetiva acerca do sistema de referenciação, cruzando estes dados com os encaminhamentos efetuados para as respetivas equipas locais de intervenção precoce. Estes resultados poderiam ajudar a identificar fragilidades e a homogeneizar procedimentos de referenciação e de resposta, promovendo a articulação entre serviços e tornando o processo mais eficiente.

Os autores consideram que este estudo acrescentou, modestamente, conhecimento acerca do processo de referenciação dos cuidados de saúde primários de crianças com problemas de comunicação e linguagem, uma vez que foi possível obter algumas perceções pertinentes dos médicos que participaram. Pensa-se que este conhecimento é fundamental para a construção de um sistema de referenciação mais compreensivo.

\section{AGRADECIMENTOS}

Agradecemos ao Dr. Cláudio Martins (USF Arco do Prado, Vila Nova de Gaia), à Dra. Catarina Machado (USF Nova Salus, Vila Nova de Gaia), ao Dr. Filipe Estrela (Centro de Saúde da Ribeira Grande, São Miguel) e à Dra. Joana Abreu (USF Conchas, Lisboa) pela colaboração na distribuição dos inquéritos. 


\section{REFERÊNCIAS BIBLIOGRÁFICAS}

1. Horwitz SM, Irwin JR, Briggs-Gowan MJ, Bosson Heenan JM, Mendoza J, Carter AS. Language delay in a community cohort of young children. J Am Acad Child Adolesc Psychiatry. 2003;42(8):932-40.

2. Busari JO, Weggelaar NM. How to investigate and manage the child who is slow to speak. BMJ. 2004;328(7434):272-6.

3. Nelson HD, Nygren P, Walker M, Panoscha R. Screening for speech and language delay in preschool children: systematic evidence review for the US Preventive Services Task Force. Pediatrics. 2006;117(2):e298319.

4. McLaughlin MR. Speech and language delay in children. Am Fam Physician. 2011;83(10):1183-8.

5. Van Agt $H$, Verhoeven L, Van Den Brink G, De Koning $H$. The impact on socio-emotional development and quality of life of language impairment in 8-year-old children. Dev Med Child Neurol. 2011;53(1):81-8.

6. Coutinho AP. As perturbações da aquisição e do desenvolvimento da linguagem: um estudo preliminar da prevalência, dos fatores associados e das necessidades de encaminhamento para terapia da fala em crianças de idade pré escolar no concelho de Oeiras [dissertation]. Lisboa: Escola Nacional de Saúde Pública, Universidade de Lisboa; 2012.

7. Aram DM, Ekelman BL, Nation JE. Preschoolers with language disorders: 10 years later. J Speech Hear Res. 1984;27(2):232-44.

8. St Clair MC, Pickles A, Durkin K, Conti-Ramsden G. A longitudinal study of behavioral, emotional and social difficulties in individuals with a history of specific language impairment (SLI). J Commun Disord. 2011;44(2):186-99.

9. Van Agt HM, Essink-Bot ML, van der Stege H, de Ridder-Sluiter JG, de Koning HJ. Quality of life of children with language delays. Qual Life Res. 2005;14(5):1345-55.

10. Conti-Ramsden G, Mok PL, Pickles A, Durkin K. Adolescents with a history of specific language impairment (SLI): strengths and difficulties in social, emotional and behavioral functioning. Res Dev Disabil. 2013;34(11):4161-9.

11. Thomas-Stonell N, Oddson B, Robertson B, Rosenbaum P. Predicted and observed outcomes in preschool children following speech and language treatment: parent and clinician perspectives. J Commun Disord. 2009;42(1):29-42.

12. Hamilton S. Screening for developmental delay: reliable, easy-to-use tools. J Fam Pract. 2006;55(5):415-22.

13. Bercow J. The Bercow report: a review of services for children and young people (0-19) with speech, language and communication needs. Nottingham: Department for Children, Schools and Families; 2008. ISBN 9781847752116

14. Ruben RJ. Redefining the survival of the fittest: communication disorders in the 21st century. Laryngoscope. 2000;110(2 Pt 1):241-5.

15. Van Der Schuit M, Segers E, Van Balkom H, Verhoeven L. Early language intervention for children with intellectual disabilities: a neurocognitive perspective. Res Dev Disabil. 2011;32(2):705-12.

16. Lai MC, Lombardo MV, Baron-Cohen S. Autism. Lancet. 2014;383(9920): 896-910.

17. Sices L, Feudtner C, McLaughlin J, Drotar D, Williams M. How do primary care physicians manage children with possible developmental delays? A national survey with an experimental design. Pediatrics. 2004; 113(2):274-82.
18. Castelhano J, Oliveira G. Médico de família: peça fundamental no desenvolvimento da criança [The family doctor: instrumental in the development of the child]. Rev Port Clin Geral. 2009;25(6):667-8. Portuguese

19. Jeyendra A, Rajadurai J, Chanmugam J, Trieu A, Nair S, Baskaran R, et al. Australian general practitioners' perspectives on their role in well-child health care. BMC Fam Pract. 2013;14:2.

20. Direção-Geral da Saúde. Programa nacional de saúde infantil e juvenil: norma n. ${ }^{\circ}$ 010/2013, de 31/05/2013. Lisboa: DGS; 2013.

21. Decreto-Lei n. ${ }^{\circ}$ 281/2009, de 6 de outubro. Diário da República. $1^{\text {a }}$ série(193):7298-301.

22. Silverstein M, Sand N, Glascoe FP, Gupta VB, Tonniges TP, O'Connor KG. Pediatrician practices regarding referral to early intervention services: is an established diagnosis important? Ambul Pediatr. 2006;6(2): $105-9$.

23. Peixoto V. Perturbações da comunicação: a importância da detecção precoce. Porto: Edições Universidade Fernando Pessoa; 2007. ISBN 9789728830915

24. Franco V, Apolónio A. Avaliação diagnóstica em intervenção precoce. Int J Dev Educ Psychol. 2011;5(1):85-94.

25. Peixoto MC, Apolónio A, Franco V. Elegibilidade, caracterização e diagnóstico nas equipas de intervenção precoce do Alentejo. Int J Dev Educ Psychol. 2011;1(1):207-18.

26. Santos MI, Coelho I, Rosário F, Machado P, Nery L, Ribeiro J, et al. Referenciação aos cuidados de saúde secundários em idade pediátrica [An analysis of referrals from primary care to a pediatric hospital outpatient clinic]. Rev Port Clin Geral. 2011;27(5):422-32. Portuguese

27. Jimenez ME, Fiks AG, Shah LR, Gerdes M, Ni AY, Pati S, et al. Factors associated with early intervention referral and evaluation: a mixed methods analysis. Acad Pediatr. 2014;14(3):315-23.

28. Hill MM, Hill A. Investigação por questionário. Lisboa: Sílabo; 2000.

29. Senecky Y, Inbar D, Diamond G, Grossman Z, Apter A, Kahan E. Pediatricians' satisfaction with their abilities to care for children with developmental, behavioral and psychosocial problems. Pediatr Int. 2007;49 (4):472-8.

30. Laraque $D$, Adams $R$, Steinbaum D, Zuckerbrot R, Schonfeld D, Jensen PS, et al. Reported physician skills in the management of children's mental health problems following an educational intervention. Acad Pediatr. 2009;9(3):164-71.

31. Horwitz SM, Caspary G, Storfer-Isser A, Singh M, Fremont W, Golzari $M$, et al. Is developmental and behavioral pediatrics training related to perceived responsibility for treating mental health problems? Acad Pediatr. 2010;10(4):252-9.

32. Sices L, Feudtner C, McLaughlin J, Drotar D, Williams M. How do primary care physicians identify young children with developmental delays? A national survey. J Dev Behav Pediatr. 2003;24(6):409-17.

33. Tuominen-Eriksson AM, Svensson Y, Gunnarsson RK. Children with disabilities are often misdiagnosed initially and children with neuropsychiatric disorders are referred to adequate resources 30 months later than children with other disabilities. J Autism Dev Disord. 2013;43(3): 579-84.

34. McConachie H, Diggle T. Parent implemented early intervention for young children with autism spectrum disorder: a systematic review. J Eval Clin Prac. 2007;13(1):120-9. 
35. Paul R. Interventions to improve communication in autism. Child Adolesc Psychiatr Clin N Am. 2008;17(4):835-56.

36. Gwynne K, Blick BA, Duffy GM. Pilot evaluation of an early intervention programme for children at risk. J Paediatr Child Health. 2009;45(3): 118-24.

37. Hollo A, Wehby JH, Oliver RM. Unidentified language deficits in children with emotional and behavioral disorders: a meta-analysis. Except Child. 2014;80(2):169-86.

38. Honigfeld L, Chandhok L, Spiegelman K. Engaging pediatricians in developmental screening: the effectiveness of academic detailing. J Autism Dev Disord. 2012;42(6):1175-82.

39. Glascoe FP. Can clinical judgment detect children with speech-language problems? Pediatrics. 1991;87(3):317-22.

40. Leung AK, Kao CP. Evaluation and management of the child with speech delay. Am Fam Physician. 1999;59(11):3121-8.

41. Comley L, Mousmanis P. Improving the odds: healthy child development. Focus on the early years: neuroscience and implications for clinical practice. Toronto: Ontario College of Family Physician; 2010.

42. Morelli DL, Pati S, Butler A, Blum NJ, Gerdes M, Pinto-Martin J, et al. Challenges to implementation of developmental screening in urban primary care: a mixed methods study. BMC Pediatr. 2014;14:16.

43. Maia AR, Fernandes J, Leite MF, Santos H, Pereira SA. Avaliação do desenvolvimento psicomotor pelos médicos de família: estudo observacional [Evaluation of psychomotor development in children by family doctors: na observational study]. Rev Port Med Geral Fam. 2016;32(4): 248-56. Portuguese

44. Sices L. Developmental screening in primary care: the effectiveness of current practice and recommendations for improvement [homepage]. Boston: The Commonwealth Fund; 2007 [cited 201823 Jul]. Available from: https://www.commonwealthfund.org/publications/fund-reports/2007/dec/developmental-screening-primary-care-effectivenesscurrent

\section{CONFLITO DE INTERESSES}

Os autores declaram não ter quaisquer conflitos de interesse.

\section{FINANCIAMENTO}

A autora responsável pela correspondência declara que este trabalho não foi objeto de qualquer tipo de financiamento externo.

\section{ENDEREÇO PARA CORRESPONDÊNCIA}

Inês Dinis

E-mail: ines.s.dinis@gmail.com

https://orcid.org/0000-0003-0554-2822

Recebido em 26-07-2018

Aceite para publicação em 19-12-2019

\section{ABSTRACT \\ PORTUGUESE FAMILY PHYSICIANS VIEWS ON THE REFERRAL OF CHILDREN WITH LANGUAGE AND COMMUNICATION PROBLEMS}

Objectives: To understand Portuguese family physicians' profile on the referral process of children with language and communication problems and to know what barriers they identify to it.

Type of study: Observational, transversal and descriptive.

Local: Central Region, Lisbon and Tejo Valley, North Region and Autonomic Region of Azores

Population: Family physicians of Central region, Lisbon, and Tejo Valley, North region and Autonomic Region of Azores Methods: An online survey was designed with 35 Likert (0-5) questions and a semi-open one. It was then sent to family physicians in Portugal according to a non-probabilistic sampling method. Ethical e confidential constraints were guaranteed.

Results: Fifty-five family physicians participated. About $40 \%$ of these physicians told they were substantially satisfied with the referral process; $55 \%$ said they were not satisfied or not very satisfied with knowledge acquired about language and communication development during medicine course; $58 \%$ of them told referrals to the Nacional Early Intervention System in Infancy occurs seldom or never. Moreover, six barriers to the referral process were identified: service constraints; lack of human resources; bureaucracy; slow process; lack of knowledge about the process; parent attitudes.

Conclusions: The results of this study do not allow us to fully describe the Portuguese family physicians' referral profile of children with language and communication problems. However, some trends in this process were identified, as well as major barriers.

Keywords: Speech and language disorders; Referral process; Family physician; Early intervention; Speech and language therapist. 